\title{
Clipping treatment of posterior communicating artery aneurysms associated with arteriosclerosis and calcification: A single center study of 136 cases
}

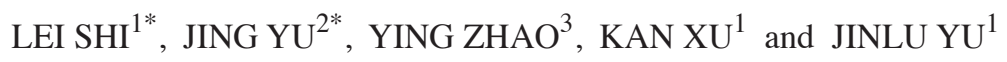 \\ Departments of ${ }^{1}$ Neurosurgery, ${ }^{2}$ Operation and ${ }^{3}$ Training, The First Hospital of Jilin University, \\ Changchun, Jilin 130021, P.R. China
}

Received May 31, 2017; Accepted October 13, 2017

DOI: $10.3892 /$ etm.2017.5525

\begin{abstract}
It is widely acknowledged that arteriosclerosis and calcification of the parent artery and aneurysm neck make it difficult to clip posterior communicating artery (PCoA) aneurysms. A total of 136 cases of PCoA aneurysms accompanied by arteriosclerosis and calcification were collected and treated with clipping in the present study. Of the 136 patients, 112 were females $(82.4 \%)$ and 24 were males (17.6\%), with ages ranging from 37 to 76 years (mean age, 60.2 years). Rupture of a PCoA aneurysm was identified in 132 cases (97.1\%), and there were 4 cases of unruptured PCoA aneurysms (2.9\%). According to the severity of arteriosclerosis and calcification, the aneurysms were divided into type I, II or III. The treatment of type I aneurysms achieved the best curative effect. It is difficult to temporarily occlude type II and III aneurysms during surgery, and temporary occlusion failed in almost $50 \%$ of cases. Types II and III were prone to intraoperative aneurysm ruptures. A significantly higher rate of intraoperative aneurysm rupture was seen in type III compared with type II cases. Type II and III cases were more likely to be treated using a fenestrated clip for aneurysm clipping compared with type I cases, and fenestrated clips were used significantly more frequently in type III cases compared with type II cases. Arteriosclerosis and calcification were likely to affect the prognosis of patients, particularly in cases with type III arteriosclerosis and calcification of the parent artery and aneurysm neck. Therefore, the stratification of the arteriosclerosis and calcification of the parent artery and aneurysm neck into
\end{abstract}

Correspondence to: Professor Jinlu Yu, Department of Neurosurgery, The First Hospital of Jilin University, 71 Xinmin Avenue, Changchun, Jilin 130021, P.R. China

E-mail: jlyu@jlu.edu.cn

*Contributed equally

Key words: arteriosclerosis, calcification, posterior communicating artery aneurysm, clipping treatment, prognosis types I-III can guide the intraoperative aneurysm clipping strategy, aid in choosing the correct clips, and inform predictions of the occurrence of rupture and hemorrhage, as well as the prognosis for aneurysms.

\section{Introduction}

Although the endovascular treatment of intracranial aneurysms has become a popular technique of surgical clipping for 25 years, coil embolization still has its limitations. In particular, the recurrence is high in cases of wide-neck, large aneurysm (1). Therefore, clipping is still an effective method for the treatment of intracranial aneurysms (2). The successful implementation of aneurysm clipping is affected by a number of factors, including the size and location of the aneurysms (3). One method that may be used to treat intracranial small aneurysms is with simple surgical clipping, as, due to the sufficient operative field, the middle cerebral artery and anterior communicating artery aneurysms may be clipped with little difficulty (4). In addition to the size and location of the aneurysms, the outcome is also associated with the extent of arteriosclerosis and calcification of the parent artery and aneurysm neck $(5,6)$. When aneurysms occur in hardened and calcified arteries, aneurysm clipping is difficult, particularly in cases of aneurysms of the posterior communicating artery (PCoA), due to their low position and wide neck $(6,7)$. The hard, calcified aneurysm neck frequently may not allow for complete closure of the clip blades, which may therefore require the use of the aneurysm clip compression technique (8). However, to the best of our knowledge, no studies have analyzed the influence of arteriosclerosis and calcification on the surgical treatment of PCoA aneurysms. The present study retrospectively analyzed 136 cases of PCoA aneurysms associated with arteriosclerosis and calcification that were treated in the Department of Neurosurgery, The First Hospital of Jilin University (Changchun, China). All patients were classified according to the extent of arteriosclerosis and calcification, and the difficulties and characteristics of treatment were analyzed for each type. The aim of the present study was to identify strategies that improve the outcomes of PCoA aneurysm clipping when arteriosclerosis and calcification are involved. 


\section{Patients and methods}

Patients. A total of 136 patients with PCoA aneurysms associated with arteriosclerosis and calcification, who were admitted to the Department of Neurosurgery, the First Hospital of Jilin University from January 2013 to June 2016, were continuously collected in this study. Written informed consent was obtained from the study participants. The study was approved by the Ethics Committee of the First Hospital of Jilin University and was conducted in accordance with the Declaration of Helsinki.

Of the 136 patients, 112 were female $(82.4 \%)$ and 24 were male $(17.6 \%)$, with ages ranging from 37 to 76 years (mean age, 60.2 years). A ruptured PCoA aneurysm was identified in 132 cases $(97.1 \%)$, and 4 patients with unruptured aneurysms (2.9\%) suffered from oculomotor palsy paralysis. According to Hunt-Hess grades (a minor revision of the Hunt and Hess scale) (9), there were 10 cases of grade I (7.4\%), 122 cases of grade II $(89.7 \%)$, and 4 cases of grade III (2.9\%) aneurysms preoperatively and 5 cases of grade I (3.7\%), 116 cases of grade II $(85.3 \%), 11$ cases of grade III $(8.1 \%)$ and 4 cases of grade IV $(2.9 \%)$ aneurysms postoperatively. Post-onset computed tomography (CT), CT angiography (CTA) and/or digital subtraction angiography examinations were performed, which confirmed diagnoses of PCoA aneurysms.

Classification of arteriosclerosis and calcification. Arteriosclerosis and calcification of the aneurysm neck and/or parent artery was identified in 136 cases of PCoA aneurysms, which was determined using operative microscopy (OPMI PENTERO 800; Zeiss GmbH, Jena, Germany). Yellow or white areas on the aneurysm neck or parent artery indicated the presence of arteriosclerosis and calcification. The parent artery and aneurysm neck were pressed during surgery. If the parent artery and aneurysm neck were hardened, they were judged to be affected by arteriosclerosis and calcification.

The parent artery and aneurysm neck were classified into three types according to the extent of arteriosclerosis and calcification: Type I, arteriosclerosis and calcification of the aneurysm neck; type II, arteriosclerosis and calcification of the parent artery; and type III, arteriosclerosis and calcification of the parent artery and aneurysm neck. Representative images of these classifications are presented in Fig. 1.

Measurement of aneurysm size. Aneurysm size was calculated using mean values of its length and width with the following formula: (Maximal length + maximal width)/2. For each aneurysm several length and width measurements were taken to obtain the accurate value.

Aneurysm clipping. The surgery adopted a pterion or enlarged pterion approach to isolate the lateral fissure, carotid cistern and part of the post-chiasmatic exposure of the aneurysm body and neck. If the anterior clinoid process occluded the aneurysm, it was resected epidurally or intradurally. The parent artery was temporarily occluded before clipping the parent artery, and the aneurysm was clipped with straight, curved, bent, bayonet-shaped or fenestrated aneurysm clips according to the location, direction and aneurysm size. As many aneurysms as possible were clipped. If a rupture occurred during aneurysm clipping, hemorrhaging was stopped using multiple aneurysm clips if necessary. If the hemorrhage could not be stopped, multiple fenestrated clips were used for aneurysm clipping. Careful attention was paid intraoperatively to protect the PCoA. Typical cases of clipping treatments are presented in Figs. 2 and 3.

Postoperative management. All patients were administered routine treatment postoperatively. If there was serious subarachnoid hemorrhaging, lumbar cistern drainage of the cerebrospinal fluid was performed. If there was a serious vasospasm, expansion and improvement of microcirculation were performed via $3 \mathrm{H}$ treatment (hypervolemia, hemodilution, hypertension) as described previously (10), while symptomatic treatment with nimodipine (Bayer AG, Leverkusen, Germany) was also provided. A 3- to 6-month CTA follow-up was performed to observe whether the aneurysm was completely occluded. According to a telephone follow-up, the prognosis was determined using the Glasgow Outcome Scale (GOS) score (11).

Statistical analysis. SPSS 19.0 statistical software (IBM Corp., Armonk, NY, USA) was used for data processing. Chi-square tests were used to analyze count data, while t-tests and one-way analysis of variance with Tukey's post hoc test were used to analyze measurement data. Data are presented as the mean \pm standard deviation and $\mathrm{P}<0.05$ was considered to indicate a statistically significant difference.

\section{Results}

Classification of arteriosclerosis and calcification. According to the classification of arteriosclerosis and calcification, 3 types were used, among the 136 cases of PCoA aneurysms, 4 were type I (3.0\%), 86 were type II $(63.2 \%)$ and 46 were type III $(33.8 \%)$.

Aneurysm size. The aneurysm diameters ranged from 1.7 to $11.8 \mathrm{~mm}$ (mean, $5.9 \pm 2.5 \mathrm{~mm}$; type I mean, $6.2 \pm 3.5 \mathrm{~mm}$; type II mean, $5.8 \pm 2.5 \mathrm{~mm}$; type III mean, $6.0 \pm 2.4 \mathrm{~mm}$; Table I). No statistically significant differences in diameter were observed among type I and II aneurysms ( $\mathrm{P}>0.05)$, type II and III aneurysms $(\mathrm{P}>0.05)$ or type I and III aneurysms ( $\mathrm{P}>0.05$; Table II).

Aneurysm clipping procedures. The anterior clinoid process was resected epidurally or intradurally before aneurysm clipping to reveal the presence of the parent artery and aneurysm neck in 2 cases. Among the 136 cases, 131 cases involved single PCoA aneurysms (96.3\%) and 5 cases involved multiple aneurysms in other locations (3.7\%), which were clipped at the same time. An aneurysm clip was used in 114 cases $(83.8 \%)$, and $\geq 2$ aneurysm clips were used for occlusion in 22 cases (16.2\%) of PCoA aneurysms.

An attempt to temporarily block the parent artery was always made in the process of aneurysm clipping. The temporary occlusion of the parent artery was successfully performed in the 4 type I cases, 41 of the 86 type II cases (47.7\%) and 22 of the 46 type III cases (47.8\%). No statistically significant difference was observed between type II and III in the success rate of temporary occlusion of the parent artery (P>0.05; Table III). 
Table I. Aneurysm diameters.

\begin{tabular}{lcccc}
\hline Parameter & Total & Type I & Type II & Type III \\
\hline Number & 136 & 4 & 86 & 46 \\
Diameter (mm) & $5.9 \pm 2.5$ & $6.2 \pm 3.5$ & $5.8 \pm 2.5$ & $6.0 \pm 2.4$ \\
\hline
\end{tabular}

Table II. Statistical comparisons among groups.

\begin{tabular}{lc}
\hline Comparison & P-value \\
\hline Type I vs. type II & 0.95 \\
Type II vs. type III & 0.93 \\
Type I vs. type III & 0.99 \\
\hline
\end{tabular}

Groups were compared using one-way analysis of variance with Tukey's post hoc test.

Intraoperative aneurysm ruptures. No intraoperative aneurysm ruptures were observed in the 4 cases of type I arteriosclerosis and calcification; among the 86 type II cases, intraoperative rupture occurred in 5 cases (5.8\%), and among the 46 type III cases, intraoperative rupture occurred in 11 cases $(23.9 \%)$. The incidence of intraoperative rupture was significantly higher in type III cases compared with cases $(\mathrm{P}<0.05$; Table III).

Clip types. Fenestrated clips were not used in the 4 type I cases during aneurysm clipping. Among the 86 type II cases, fenestrated clips were used in 11 cases (12.8\%), and among the 46 type III cases, fenestrated clips were used in 13 cases (28.3\%). The use of fenestrated aneurysm clips was significantly more common in type III cases compared with type II cases $(\mathrm{P}<0.05$; Table III).

Postoperative outcomes. After aneurysm clipping, the 4 type I cases did not exhibit any complications. Among the 86 type II cases, 9 cases (10.5\%) deteriorated postoperatively, as the Hunt-Hess grades increased, and among the 46 type III cases, $11(23.9 \%)$ deteriorated postoperatively. A significantly higher proportion of patients deteriorated postoperatively among the type III cases compared with the type II cases $(\mathrm{P}<0.05$; Table III).

CTA follow-up was performed at 3-6 months postoperatively. Among the 136 cases, 120 cases (88.2\%) were followed up with CTA. The results indicated that the aneurysms were completely closed without recurrence. At 6-12 months, a telephone follow-up was performed for the majority of patients $(132 / 136,97.1 \%)$. The GOS scores were as follows: 126 patients $(95.5 \%)$ achieved a score of 5 ; 4 patients $(3 \%)$ achieved a score of 4 ; and 2 patients $(1.5 \%)$ achieved a score of 3 .

\section{Discussion}

The present study collected data from 136 patients, $82.4 \%$ of whom were female, generating a female-to-male ratio of 4.7:1. However, the female-to-male incidence ratio of intracranial ruptured aneurysms was between 1:1 and 2:1. In a 2001 study in Japan by Osawa et al (12), the female-to-male onset ratio was 1.46:1, and the ratio in the Taiwan region of China has been reported to be 1.47:1 (13). Given the higher female-to-male ratio in the present cohort, this may have resulted in a gender-bias compared with these previous studies. Therefore, the female-to-male ratio in patients with PCoA aneurysms associated with arteriosclerosis and calcification may be markedly different from that of the general population. Accordingly, the female-to-male ratio was similar to that of intracranial aneurysms with wall and neck calcification. For instance, Kizilkilic et al (14) studied a total of 103 patients (74\% female) in 2016 with wall and neck calcification using flat-detector CT.

Currently, two primary surgical treatments are used for intracranial aneurysms, namely, neurosurgical clipping and endovascular coiling (2). The International Subarachnoid Aneurysm Trial in 2015 indicated that these two methods were effective for the treatment of intracranial aneurysms (15). Currently, the surgical clipping of intracranial aneurysms is a popular treatment (16). Neurosurgical clipping can be affected by numerous factors, including the age and sex of the patient, the location and size of the aneurysm, and the extent of calcification or sclerosis of the aneurysm and parent artery (14). Among these factors, arteriosclerosis and calcification of the aneurysm and parent artery are particularly important. Intracranial PCoA aneurysms are often more affected by arteriosclerosis and calcification than aneurysms located in other sites, such as anterior communicating artery and middle cerebral artery, primarily due to the PCoA aneurysms originating from the internal carotid artery at the entrance to the skull, as this intracranial segment is more likely to exhibit arteriosclerosis and calcification $(7,17)$.

It was identified that if the arteriosclerosis and calcification of affected PCoA aneurysms was more severe, there are increased risks associated with clipping surgeries, such as intraoperative rupture, incomplete clipping (8). This suggested that different degrees of calcification of the aneurysm and parent artery had different influences on surgery, with more severe arteriosclerosis and calcification resulting in more difficult clipping surgery The cases in the present study were classified as follows: Type I, arteriosclerosis and calcification of the aneurysm neck; type II, arteriosclerosis and calcification of the parent artery; or type III, arteriosclerosis and calcification of the aneurysm neck and parent artery. During the treatment of patients in the present study, it was identified that this classification of PCoA aneurysms could guide the surgical clipping treatment and provide some insight into prognosis.

The success rate of temporary occlusion during aneurysm clipping was different among patients with different degrees of arteriosclerosis and calcification. For intracranial aneurysm clipping, the parent artery should be temporarily occluded before clipping to reduce the pressure caused by blood flow within the aneurysm, thereby reducing the risk of rupture and facilitating a more completely occluded aneurysm during aneurysm clipping surgery $(18,19)$. However, when the arteriosclerosis or calcification of the parent artery is more serious, the strength of temporary aneurysm clips may not be sufficient, and forced clipping of aneurysms may cause damage of the parent arteries or rupture of the endometrial 
Table III. Surgical results for patients with type II and III arteriosclerosis and calcification.

\begin{tabular}{lrrrrrr}
\hline & \multicolumn{2}{c}{ Type II } & & \multicolumn{2}{c}{ Type III } & \\
\cline { 2 - 3 } \cline { 5 - 6 } Result & $\mathrm{n}$ & & & $\mathrm{n}$ & $\%$ & P-value \\
\hline Temporary occlusion & 41 & 47.7 & & 22 & 47.8 & 0.987 \\
Intraoperative rupture & 5 & 5.8 & & 11 & 23.9 & 0.040 \\
Use of fenestrated clip & 11 & 12.8 & & 13 & 28.3 & 0.028 \\
Postoperative aggravation & 9 & 10.5 & 11 & 23.9 & 0.040 \\
\hline
\end{tabular}

Groups were compared using t-tests.
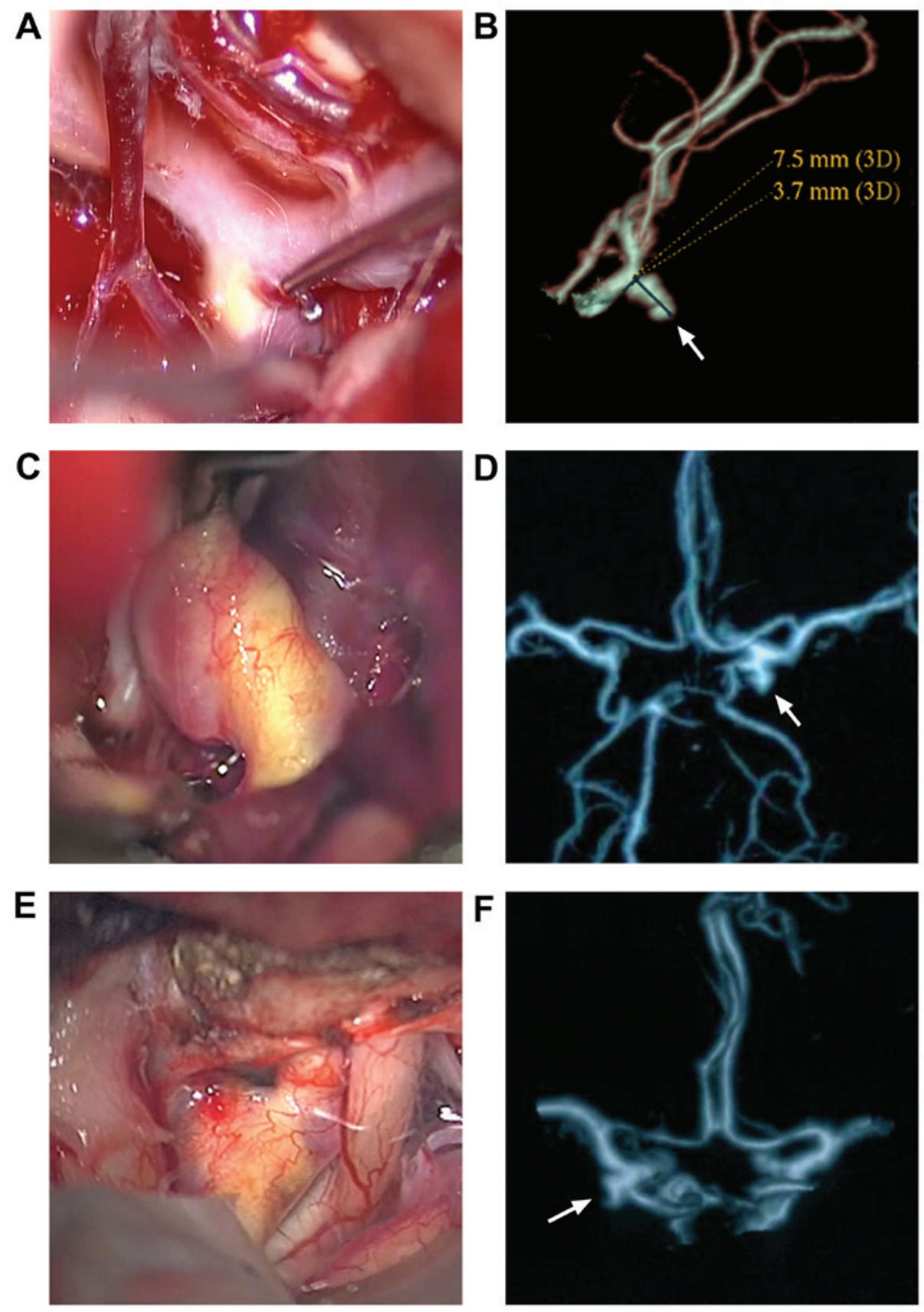

Figure 1. Classification of arteriosclerosis and calcification. (A) Type I, only the aneurysm neck exhibits arteriosclerosis and calcification in intraoperative view. (B) CTA reconstruction indicating the PCoA aneurysm (arrow). (C) Type II, only the parent artery (internal carotid artery) exhibits arteriosclerosis and calcification. (D) CTA reconstruction indicating the PCoA aneurysm (arrow). (E) Type III, both the parent artery and aneurysm neck exhibit arteriosclerosis and calcification. (F) CTA reconstruction indicating the PCoA aneurysm (arrow). CTA, CT angiography; PCoA aneurysm, posterior communicating artery aneurysm. 

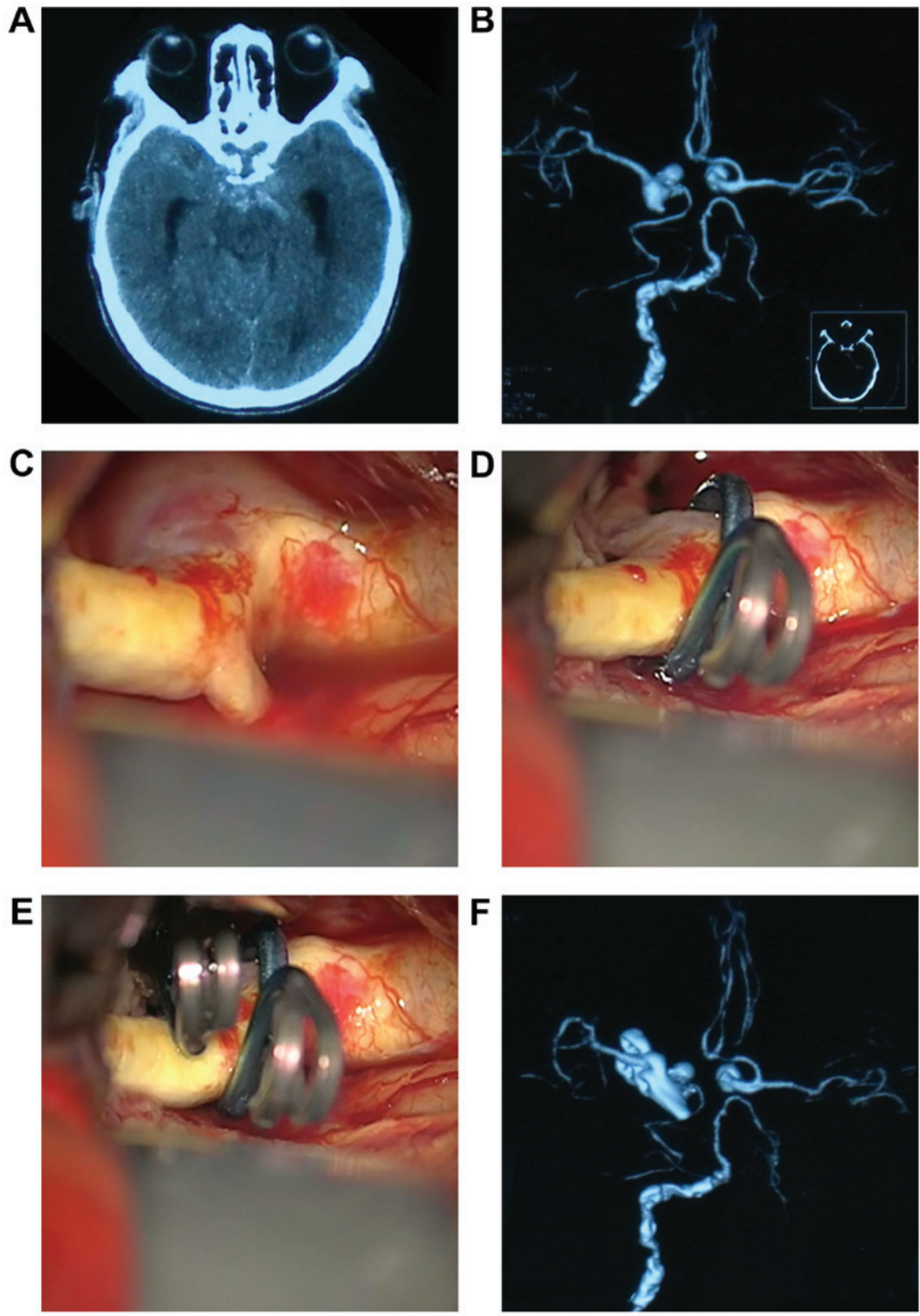

Figure 2. Images of aneurysm clipping procedures from the same patient in case 1. (A) CT scan indicating subarachnoid hemorrhage. (B) Preoperative CTA showing a left PCOA aneurysm. (C) Intraoperative imaging indicating obvious arteriosclerosis and calcification of the parent arteries. (D) Use of the first fenestrated clip to clip the PCoA aneurysm. (E) Use of the second straight clip to clip the PCoA aneurysm. (F) Postoperative CTA indicating complete clipping of aneurysm. CT, computed tomography; CTA, CT angiography; PCoA aneurysm, posterior communicating artery aneurysm.

plaque (20). In the present study, for type I cases, since only the aneurysm neck exhibited arteriosclerosis or calcification, temporarily occluding the parent arteries was not difficult. However, for type II and III cases, since the parent arteries exhibited arteriosclerosis and calcification, the failure rate of temporary occlusion was almost $50 \%$. However, there was no statistically significant difference between type II and III cases in the success rate of temporary occlusion because these two types had the same extent of arteriosclerosis and calcification of the parent artery.

The rupture and hemorrhage occurring in the process of clipping PCoA aneurysms has notable consequences because PCoA aneurysms originate from the internal carotid artery just entering the skull and experience a high shear force $(21,22)$.
In addition, PCoA aneurysms are often wide-necked, and arteriosclerosis and calcification increase the risk of rupture and hemorrhage $(23,24)$. The occurrence of intraoperative rupture is also likely to be associated with the position and degree of arteriosclerosis and/or calcification. In the present study, it was identified that for type I PCoA aneurysms, although the aneurysm neck was hardened or calcified, aneurysm clipping was still safe due to the good flexibility of the normal parent artery. The arteriosclerosis and calcification of the parent artery in type II and III cases easily led to intraoperative rupture during aneurysm clipping, with type III aneurysms having a greater risk of intraoperative rupture than type II because the aneurysm neck and parent artery exhibited arteriosclerosis or calcification. 

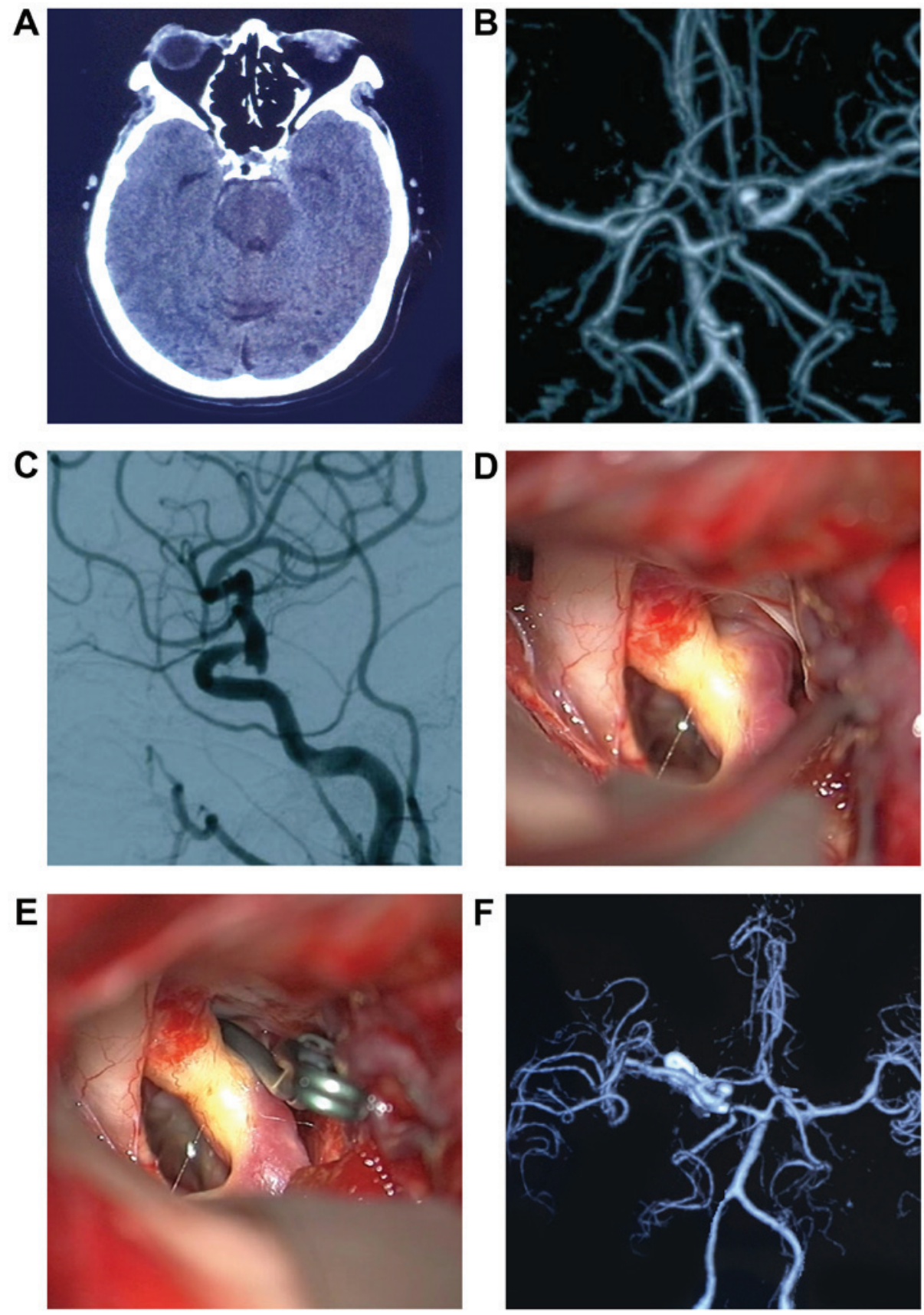

Figure 3. Images of aneurysm clipping procedures from the same patient in case 2. (A) CT scan indicating subarachnoid hemorrhage. (B) Preoperative CTA indicating right PCoA aneurysm. (C) Preoperative digital subtraction angiography indicating PCoA aneurysm. (D) Intraoperative imaging indicating obvious arteriosclerosis and calcification of the parent arteries. (E) Use of a curved clip in aneurysm clipping. (F) Postoperative CTA indicating the complete clipping of aneurysm. CT, computed tomography; CTA, CT angiography; PCoA aneurysm, posterior communicating artery aneurysm.

There are many techniques for clipping PCoA aneurysms, with $>100$ types of intracranial aneurysm clips available (25). Among them, fenestrated clips for PCoA aneurysm clipping are occasionally used (26). The use of straight, curved or angled clips for clipping aneurysm necks and parent arteries with arteriosclerosis and calcification can easily cause intraoperative rupture and hemorrhage due to the low flexibility of these structures (27). To prevent this, the application of fenestrated clips over hardened and calcified aneurysm necks and parent arteries is a good alternative because fenestrated clips can avoid severely shrinking the parent artery and aneurysm neck (8). In the present study, fenestrated clips were not used for Type I PCoA aneurysms. Certain cases of type II and III aneurysms were treated with fenestrated clips, and this was more frequent in type III cases than for type II cases.

For PCoA aneurysm clipping surgeries involving arteriosclerosis and calcification, the prognosis of patients is closely associated with the classification of arteriosclerosis and calcification $(8,28)$. Extensive arteriosclerosis and calcification will result in a poor outcome. For instance, in the present study, $10.5 \%$ of type II patients deteriorated after aneurysm clipping, and $23.9 \%$ of type III patients deteriorated after aneurysm clipping. This difference between types II and III was significant.

Aside from the use of clips to treat patients with type II and III aneurysms, endovascular coiling embolization is known to be effective for treating patients with these types of 
aneurysms (29). Use of the coiling technique to treat aneurysms with arteriosclerosis and calcification in the parent artery and aneurysm neck does not significantly change the aneurysm shape (30). Therefore, coiling is an alternative treatment for PCoA aneurysms accompanied by arteriosclerosis and calcification (31).

In conclusion, 136 cases of clipping surgeries for PCoA aneurysms accompanied by arteriosclerosis and calcification were described in the present study. It was identified that classification of the extent of arteriosclerosis and calcification of the parent artery and aneurysm neck into types I-III can guide the aneurysm clipping operation strategy, assist with choosing the clips and help in predicting the occurrence of rupture and hemorrhaging. It can also provide some insight into the prognosis for intraoperative aneurysms.

\section{References}

1. Kim SO, Chung YG, Won YS and Rho MH: Delayed ischemic stroke after flow diversion of large posterior communicating artery aneurysm. J Cerebrovasc Endovasc Neurosurg 18: 19-26, 2016.

2. Spetzler RF, Zabramski JM, McDougall CG, Albuquerque FC, Hills NK, Wallace RC and Nakaji P: Analysis of saccular aneurysms in the barrow ruptured aneurysm trial. J Neurosurg: Feb 24, 2017 (Epub ahead of print).

3. Acciarri N, Toniato G, Raabe A and Lanzino G: Clipping techniques in cerebral aneurysm surgery. J Neurosurg Sci 60: 83-94, 2016.

4. Matano F, Mizunari T, Kominami S, Suzuki M, Fujiki Y, Kubota A, Kobayashi S, Murai Y and Morita A: Retrograde suction decompression of a large internal carotid aneurysm using a balloon guide catheter combined with a blood-returning circuit and STA-MCA bypass: A technical note. Neurosurg Rev 40: 351-355, 2017.

5. van den Wijngaard IR, Holswilder G, van Walderveen MA, Algra A, Wermer MJ, Zaidat OO and Boiten J: Treatment and imaging of intracranial atherosclerotic stenosis: Current perspectives and future directions. Brain Behav 6: e00536, 2016.

6. Kockelkoren R, Vos A, Van Hecke W, Vink A, Bleys RL, Verdoorn D, Mali WP, Hendrikse J, Koek HL, de Jong PA and De Vis JB: Computed tomographic distinction of intimal and medial calcification in the intracranial internal carotid artery. PLoS One 12: e0168360, 2017

7. Subedi D, Zishan US, Chappell F, Gregoriades ML, Sudlow C, Sellar R and Wardlaw J: Intracranial carotid calcification on cranial computed tomography: Visual scoring methods, semiautomated scores, and volume measurements in patients with stroke. Stroke 46: 2504-2509, 2015.

8. Inci S, Akbay A and Orunoglu M: Aneurysm clip compression technique in the surgery of aneurysms with hard/calcified neck. World Neurosurg 84: 688-696, 2015.

9. Stranjalis G and Sakas DE: A minor revision of Hunt and Hess scale. Stroke 32: 2208, 2001.

10. Chittiboina P, Conrad S, McCarthy P, Nanda A and Guthikonda B: The evolving role of hemodilution in treatment of cerebral vasospasm: A historical perspective. World Neurosurg 75: 660-664, 2011.

11. Dean JM and Kaufman ND: Prognostic indicators in pediatric near-drowning: The Glasgow coma scale. Crit Care Med 9: $536-539,1981$.

12. Osawa M, Hongo K, Tanaka Y, Nakamura Y, Kitazawa K and Kobayashi S: Results of direct surgery for aneurysmal subarachnoid haemorrhage: Outcome of 2055 patients who underwent direct aneurysm surgery and profile of ruptured intracranial aneurysms. Acta Neurochir (Wien) 143: 655-664, 2001.

13. Howng SL, Hung TP, Kwan AL and Lin CL: Intracranial aneurysm in Taiwan. J Formos Med Assoc 94 (Suppl 2): S73-S80, 1995 (In Chinese).
14. Kizilkilic O, Huseynov E, Kandemirli SG, Kocer N and Islak C: Detection of wall and neck calcification of unruptured intracranial aneurysms with flat-detector computed tomography. Interv Neuroradiol 22: 293-298, 2016.

15. Molyneux AJ, Birks J, Clarke A, Sneade M and Kerr RS: The durability of endovascular coiling versus neurosurgical clipping of ruptured cerebral aneurysms: 18 year follow-up of the UK cohort of the International Subarachnoid Aneurysm Trial (ISAT). Lancet 385: 691-697, 2015.

16. Spetzler RF, McDougall CG, Zabramski JM, Albuquerque FC, Hills NK, Russin JJ, Partovi S, Nakaji P and Wallace RC: The barrow ruptured aneurysm trial: 6-year results. J Neurosurg 123: 609-617, 2015

17. Kim KM, Kang HS, Lee WJ, Cho YD, Kim JE and Han MH: Clinical significance of the circle of Willis in intracranial atherosclerotic stenosis. J Neurointerv Surg 8: 251-255, 2016.

18. Pereira BJ, Holanda VM, Giudicissi-Filho M, Borba LA, de Holanda CV and de Oliveira JG: Assessment of cerebral blood flow with micro-doppler vascular reduces the risk of ischemic stroke during the clipping of intracranial aneurysms. World Neurosurg 84: 1747-1751, 2015.

19. Bhatia S, Sekula RF, Quigley MR, Williams R and Ku A: Role of calcification in the outcomes of treated, unruptured, intracerebral aneurysms. Acta Neurochir (Wien) 153: 905-911, 2011.

20. Ferch R, Pasqualin A, Pinna G, Chioffi $F$ and Bricolo A: Temporary arterial occlusion in the repair of ruptured intracranial aneurysms: An analysis of risk factors for stroke. J Neurosurg 97: 836-842, 2002

21. Hsu CE, Lin TK, Lee MH, Lee ST, Chang CN, Lin CL, Hsu YH, Huang YC, Hsieh TC and Chang CJ: The impact of surgical experience on major intraoperative aneurysm rupture and their consequences on outcome: A multivariate analysis of 538 microsurgical clipping cases. PLoS One 11: e0151805, 2016.

22. Schuette AJ, Barrow DL and Cohen-Gadol AA: Strategies to minimize complications during intraoperative aneurysmal hemorrhage: A personal experience. World Neurosurg 83: 620-626, 2015.

23. Fukuda H, Hayashi K, Yoshino K, Koyama T, Lo B, Kurosaki Y and Yamagata S: Impact of aneurysm projection on intraoperative complications during surgical clipping of ruptured posterior communicating artery aneurysms. Neurosurgery 78: 381-390, 2016.

24. Takeuchi S, Tanikawa R, Goehre F, Hernesniemi J, Tsuboi T, Noda K, Miyata S, Ota N, Sakakibara F, Andrade-Barazarte H and Kamiyama H: Retrograde suction decompression for clip occlusion of internal carotid artery communicating segment aneurysms. World Neurosurg 89: 19-25, 2016.

25. Matsukawa H, Fujii M, Akaike G, Uemura A, Takahashi O, Niimi Y and Shinoda M: Morphological and clinical risk factors for posterior communicating artery aneurysm rupture. J Neurosurg 120: 104-110, 2014.

26. Ota N, Tanikawa R, Noda K, Tsuboi T, Kamiyama H and Tokuda S: The efficiency of the new Yasargil titanium fenestrated mini-clips for ideal clipping of a cerebral aneurysm. Surg Neurol Int 6 (Suppl 21): S553-S559, 2015.

27. Shi L, Yuan Y, Guo Y and Yu J: Intracranial post-embolization residual or recurrent aneurysms: Current management using surgical clipping. Interv Neuroradiol 22: 413-419, 2016.

28. Sharma M, Brown B, Madhugiri V, Cuellar-Saenz H, Sonig A, Ambekar S and Nanda A: Unruptured intracranial aneurysms: Comparison of perioperative complications, discharge disposition, outcome, and effect of calcification, between clipping and coiling: A single institution experience. Neurol India 61: 270-276, 2013.

29. Darsaut TE, Findlay JM, Magro E, Kotowski M, Roy D, Weill A, Bojanowski MW, Chaalala C, Iancu D, Lesiuk H, et al: Surgical clipping or endovascular coiling for unruptured intracranial aneurysms: A pragmatic randomised trial. J Neurol Neurosurg Psychiatry 88: 663-668, 2017.

30. Diaz O and Rangel-Castilla L: Endovascular treatment of intracranial aneurysms. Handb Clin Neurol 136: 1303-1309, 2016.

31. Zheng F, Dong Y, Xia P, Mpotsaris A, Stavrinou P, Brinker G, Goldbrunner R and Krischek B: Is clipping better than coiling in the treatment of patients with oculomotor nerve palsies induced by posterior communicating artery aneurysms? A systematic review and meta-analysis. Clin Neurol Neurosurg 153: 20-26, 2017. 